\title{
A SIP-based Device Communication Service for OSGi Framework
}

\author{
Dennis Bushmitch \\ Wanrong Lin \\ Andrzej Bieszczad \\ Alan Kaplan \\ Panasonic Technologies \\ 2 Research Way, $3^{\text {rd }}$ Floor \\ Princeton, New Jersey 08540, USA \\ db, wanrong, ajb, kaplana@research.panasonic.com
}

\author{
Vasilis Papageorgiou \\ Algirdas Pakstas \\ London Metropolitan University, 166-220 Holloway \\ Communications technology and Mathematics \\ Road, London, N7 8DB, England \\ v.papageorgiou@dsl.pipex.com \\ a.pakstas@londonmet.ac.uk
}

\begin{abstract}
This paper outlines an approach toward extending the OSGi (Open Services Gateway Initiative) framework to support interoperability with mobile devices. The approach is based on the use of SIP (Session Initiation Protocol), which is likely to be employed by nextgeneration mobile networks. The extensions to OSGi allow a SIP device to participate in an OSGi platform and allow OSGi services to be exported into a mobile (SIP-based) environment. In this paper, we propose an architecture supporting the integration of SIP with OSGi, provide some application scenarios and describe the details of the SIP Service for OSGi.
\end{abstract}

\section{Introduction}

The evolution of both mobile and home networking technologies offers opportunities for supporting the interoperation between mobile devices and devices residing in a home network. By interoperation, we mean the ability for mobile devices, such as cell phones and PDAs, to discover, connect, control and interact with devices in a home, such as PVRs, televisions and cameras.

In the mobile domain, the expected transition to an all-IP wireless infrastructure means that SIP [3] (Session Initiation Protocol) is likely to be employed by nextgeneration mobile devices. Although originally intended for session management for Voice over IP, SIP is becoming increasingly popular in other applications. These applications can be traced to SIP's support for device mobility and location independence [5], wide area service mobility and strengthened security [6]. SIP also supports event notification, which is critical for device control applications. Examples of applications based on SIP include device control [10], device/service capability negotiation [7] and instant messaging and presence [8].
In the home networking domain, various low-level home networking technologies and protocols are proliferating the market. Examples include 1394, Bluetooth, 802.11 and X10. To help manage the diversity and heterogeneity inherent in home networks, frameworks, such as OSGi (Open Services Gateway Initiative) [11], are being defined in various standard organizations. Specifically, OSGi is a Java-based framework that supports the delivery, activation and execution of services (called bundles) to home networks. All services can be managed remotely, thus allowing service providers to adapt their products to a consumer's needs while keeping these products up to date. OSGi is independent of lower-level communication protocols and provides a middleware layer that can accommodate a variety networking technologies. For example, an OSGi-compliant platform can integrate both TCP/IP- and Bluetooth- connected devices, or provide internetworking between UPnP devices and Jini devices [1],[12].

We believe that the combination of SIP and OSGi provides the necessary ingredients for supporting interoperation between mobile device and home network devices. In this paper, we propose a SIP-based device communication service for the OSGi framework. Our research in defining this service is pursuing two parallel, yet complementary, directions. One direction involves extending OSGi to handle a SIP device, while the other direction involves allowing OSGi devices' functionality to be exported into a mobile environment.

Extending OSGi to handle a SIP device facilitates communication between SIP-based devices/services and other OSGi-based services. In general, this extension allows any OSGi bundle to interact with a registered SIP device/service. As a result, a SIP device/service is able to interact with other OSGi devices/services, such as UPnP or Jini devices. For example, a SIP-based mobile phone can act as a remote control for UPnP-enabled audio/video equipment or for Jini-enabled printers, which provides the 
ability to access music and video stored on a UPnP home media server, or to print images on a Jini printer.

Allowing an OSGi device's functionality to be exported into a mobile environment means that devices and services in a home environment gain networkindependent service mobility. Bundles can selectively export OSGi devices' functionalities as enabled by various OSGi services into the mobile SIP device/service representation [2]. Because of its flexibility, SIP-based transport and eventing do not generally restrict exportation of devices functionality. Such exportation becomes strictly a function of the bundle, which implements SIP user agent and exports OSGi device functions. An example of such SIP-enabled feature exportation, bundle that communicates with local UPnP device can export its "evented" state changes into mobile domain by implementing SIP-based event notification. This further makes part of UPnP device functionality importable into other OSGi frameworks, as SIP Service allows SIP device importation.

The remainder of this paper is organized as follows. Subsections 1.1 and 1.2 provide a brief overview of OSGi and SIP. We outline some use cases that define the OSGi SIP Service design requirements in Section 2. Section 3 provides a description of the proposed SIP Service, and Section 4 elaborates the service's support for device mobility. We describe an Application Programming Interface (API) for the OSGi SIP service via examples in Section 5. Section 6 concludes with a summary and outlines our directions for future research.

\subsection{Open Service Gateway Interface (OSGi)}

OSGi [11] is a Java-based framework for delivering services to residential users over a network. Services can be configured dynamically by activating and de-activating service or application packages called bundles. Services can be managed remotely, thus allowing service providers to adapt their products to customer needs while keeping these products up to date.

The OSGi architecture is specified in terms of a collection of Java APIs, some mandatory and some optional. Providers of OSGi-compliant gateway devices must provide implementation of the mandatory APIs. Certain services are mandatory for the OSGi framework's compliance certification, so that application providers can make assumptions about service availability on any OSGi-compliant platform. This makes application bundles portable, and therefore very attractive to implement and deploy.
From the OSGi's perspective, a device may be a piece of hardware or software, as long as there exists a record of the device and its category in the OSGi device service registry. Device service is then responsible for device discovery and registration with the OSGi device registry. An integral part of the OSGi platform is the Device Manager, which is a utility that attaches proper drivers to devices, basing the driver attachment decision on the OSGi device category. The device management mechanism of OSGi provides a very flexible environment in which devices and services can be added or removed dynamically. Device services can represent various levels of abstraction. For example, a single device can be seen by various OSGi entities as a Jini device, as a printer or as a USB device.

\subsection{Session Initiation Protocol (SIP)}

SIP is a call setup and management protocol for multimedia communication [3]. SIP has several attractive features. It is simpler than earlier session initiation protocols like H.323. Present and forecasted proliferation of SIP-enabled devices [4],[9] is a direct result of featurerich context and underlying simplicity. Similarly to HTTP or SMTP, SIP is text-based and well integrated with the Internet family of protocols. SIP is an extensible protocol, and therefore very flexible in accommodating novel applications. It does not use centralized management, so it is highly scalable. SIP is an application layer protocol that is agnostic about the underlying lower layer transport protocols. That and the fact that it can be implemented on a small footprint make it very attractive for developers of devices ranging from small, networked gadgets to mobile phones to set-top-boxes to personal computers.

SIP is a client-server protocol. There are two fundamental components of the SIP architecture: the user agent (UA) and the SIP server. There are two types of user agents; usually, both reside on the same endpoint device. A User Agent Client (UAC) is responsible for sending requests on behalf of the user of the device, while a User Agent Server (UAS) responds to the requests from UAC. In the simplest configuration, two pairs of user agents can maintain communication between the two devices on which they reside. SIP servers (registrar / proxy / redirect) provide flexibility in managing the communication. Instead of targeting parties directly, UAs can instead send call setup requests to SIP proxy servers that can handle call setup on their behalf. An advantage of using a Proxy Server is that the caller does not need to know all the details of the called party's current location and availability. A special SIP URI is used for addressing. The SIP Proxy Server resolves the address with possible assistance from DNS servers and another SIP server. 
Every SIP client has to register with a Registrar, so its location and availability can be determined dynamically. The primary registration defines the home Proxy Server for that client. The reference to the home proxy is carried around with the client (or delivered by the user on demand). It is used to update the location information whenever the client registers in a new environment. The registration request may be directed to a well-known SIP multicast address although the home SIP server will often be pre-configured on the client device. Redirect Servers are similar to proxies, but instead of handling call setup on clients' behalves, they merely return the current location information back to the calling UAC using the redirection data.

Basic SIP call management is achieved with a small number of messages called methods. The initiation of a call is done using the INVITE method, which takes several parameters that determine the address of the target and the originator of the call, subject of the call, call priority and routing requests, caller preference for target location and desired features of the response. The REGISTER method allows clients to register with registrars. Other call control methods are BYE (indicates a desire to end the session in progress), ACK (acknowledges successful completion of other commands) and CANCEL (cancels all previous requests). The endpoints can use the OPTIONS method to solicit capabilities of the communication devices and other desired call parameters. The MESSAGE method can be used to transfer any information. SIP notifications are important in managing events. They also allow for implementation of advanced call management features. A client can use the SUBSCRIBE method to activate the notification mechanism about the event described in the payload of the command. Once the event occurs, the client will be made aware of it with the NOTIFY method.

SIP initially was used only for session setup and management; other protocols are used to carry on the communication. For example, Real Time Protocol (RTP) could be used to actually transfer multimedia data. However, as powerful features of SIP like eventing, security [6] and mobility have proven themselves as very useful for many applications, SIP has been gradually overloaded with functions other than session management.

\section{Use case scenarios and requirements}

The following use cases and technical requirements need to be supported by the proposed SIP Service:

1) Wide area network-based communication of SIP devices (e.g., 3GPP2, 4G mobile clients, SIP phones, remote controllers, etc.): SIP enables secure communication between wide area mobile devices and devices/services connected and registered with an OSGi gateway. By utilizing the proposed API, a bridging bundle can translate between SIP devices and other different local devices and services (e.g., UPnP, X10, 1394, etc.). The existing SIP-based networking infrastructure will be utilized.

2) OSGi device and service application-layer mobility using SIP: Selected OSGi device/service functionality can be exported as a SIP device and registered with SIP proxy/location service. This device/service then gains the mobility feature of SIP, and can move and register with another SIP server of a SIP Service-enabled OSGi gateway while maintaining its SIP service/device identity. This use case utilizes SIP-based logical naming, which preserves service portability and can also abstract service definitions from the physical devices to a capability-based functionality

3) OSGi inter-gateway bridging: Devices registered with one OSGi service registry are exported by bridging bundles as SIP devices and imported into another service registry. The bridging bundles of the second OSGi platform can then seamlessly translate these SIP devices into the functions of the desired devices category (e.g., UPnP OSGi device) Thus cross-framework device and service mobility is achieved. In an extreme example of this functionality, one OSGi gateway can gain full mobility with respect to another OSGi gateway, while maintaining device and service connectivity.

\section{SIP Service Architecture Overview}

An OSGi-compliant home gateway typically has several home networking devices registered with it. A conceptual architecture is shown in Fig. 1. The gateway-enabled devices may be using several different networking technologies, and rely on various of OSGi services and bridging bundles to enable seamless internetworking among them (e.g., UPnP OSGi service, Jini OSGi service, etc.), as shown in Fig. 2. An overview of how such crossnetwork bridging can be achieved in OSGi is given in [1]. OSGi services provide native device discovery and communication features, while registering devices with an OSGi device service registry and enabling devices' functionality via standardized APIs.

The proposed SIP Service deals with SIP-specific functions, such as registrations and messaging, as well as the OSGi-specific functions. If the SIP Service is available on an OSGi gateway deployment, it should be properly registered with the OSGi service registry and discoverable by any bundle that wishes to use it. After the OSGi platform activates the SIP Service driver, the SIP 
Service instantiates an instance of the SIP server - a valid SIP server, as defined by [3]. This server acts as a SIP registrar or a SIP proxy server for the "attached" to this OSGi platform SIP devices. The SIP server's primary role involves registering SIP devices and proxying SIP messages when such devices become mobile. A detailed description of SIP mobility is provided in Section 4.

SIP Service further registers all registered with SIP server SIP devices with the OSGi Framework device registry, assigning them a proper category as of "SIP Device". Any bundle wishing to communicate with some SIP device of interest, can access OSGi device registry, locate devices of "SIP Device" category, and then use a function of our SIP Service API to retrieve the object's handle of the corresponding to this SIP device SIPDevice object. The general hierarchy of the SIP Service API is shown in Fig. 3.

There are two distinct types of SIP devices. One type is a physical SIP device, which posses a valid SIP User Agent (UA) and is usually an independent network entity. Such UAs normally register themselves with the SIP server started by the SIP Service. The other type is a virtual SIP device, which is any OSGi executable bundle that (by utilizing the proposed SIP Service API) behaves as a valid SIP UA. Both types of SIP devices can be registered with SIP server and have valid SIP URLs. A virtual SIP device has a URL of the form: sip:bundleID@gw.home.net, while a physical SIP device has a URL of the form: sip:devManufacturerID@gw.home.net.

A SIPDevice object can reference both virtual and physical SIP devices. The SIP Service provides an additional capability for any two bundles located either on the same gateway or on two different gateways to communicate with each other in the same manner that any two SIP entities can communicate, i.e., with all implications of SIP-enabled mobility, eventing and security features. To take advantage of this mobile communication feature, a bundle needs to implement SIP UA functionality utilizing the proposed SIP Service SIPUserAgent API.

By using the SIPUserAgent interface and SIPDevice reference object, SIP protocol methods can be originated to facilitate communication between real or virtual UAs. These can be requests, acknowledgements or one-way messages, depending on the SIPUserAgent method invoked. SIPUserAgent represents a SIP agent that an application owns (i.e., the application has full control of the agent). SIPDevice objects on other hand represent references to those SIP agents registered with OSGi platform that an application bundle can access by sending SIP messages to/from via methods of a SIPUserAgent.

To translate between diverse device frameworks, socalled bridging bundles are introduced to perform application layer bridging, as shown in Fig. 4. For example, a bridging bundle between UPnP and SIP frameworks will utilize both UPnP and SIP OSGi services. The bridging bundle can instantiate UPnP and SIP device objects and translate UPnP events into SIPbased event notifications, or vise-versa. It can encapsulate UPnP SOAP control messages into SIP MESSAGE messages, with appropriate message payload and header translations. It is up to a bridging bundle to determine how much OSGi device functionality to export into a SIP domain.

As an example of the advanced functionality achieved by utilizing the proposed SIP Service, an OSGi executable bundle may choose to export the functionality of an OSGi device it has access to via SIP User Agent (UA) messaging and eventing functions. This SIP UA can then be imported as a SIP device by another SIP Serviceequipped OSGi gateway and referenced there as a SIP device (no longer virtual). This implies a very powerful function of cross-gateway device importation/exportation, with devices and services being fully mobile, and gateways acting as intermediate hosts for mobile devices.

\section{SIP Device at a Foreign Gateway: SIP Device Mobility Explained}

The proposed SIP Service architecture can handle any SIP requests/messages regardless of whether the SIP device is located locally or has moved to a new location and registered with a different SIP registrar server. If a SIP device relocates to a different domain, as outlined in Fig. 5, this SIP device should perform the following steps. After obtaining an IP address at the new location, the device has to register with the local SIP registrar (foreign to the home gateway) (step 1). The device needs to update its location information with the home SIP server (step 2) to indicate its new location. However, the home registrar server may be unaware of the exact location of the device in the visited domain. The home registrar and proxy will redirect all SIP messages to the proxy server in the visited domain (step 3). This allows for the roaming device to change its IP address if it is changing location within the sub-domains of the visited network. The presence of another OSGi gateway in a visiting domain is purely optional. The SIP Service would normally continue to send and to receive SIP messages via its SIP proxy. Both, 
pre-call and mid-call SIP terminal mobility can be supported [5].

\section{Application Program Interface}

The following Java code illustrates the use of SIP Service by an OSGi bundle. In this example, the user instantiates SIPUserAgent, retrieves a SIPDevice reference object from the SIPServer, and then uses it to send some device control message to the corresponding SIP device.

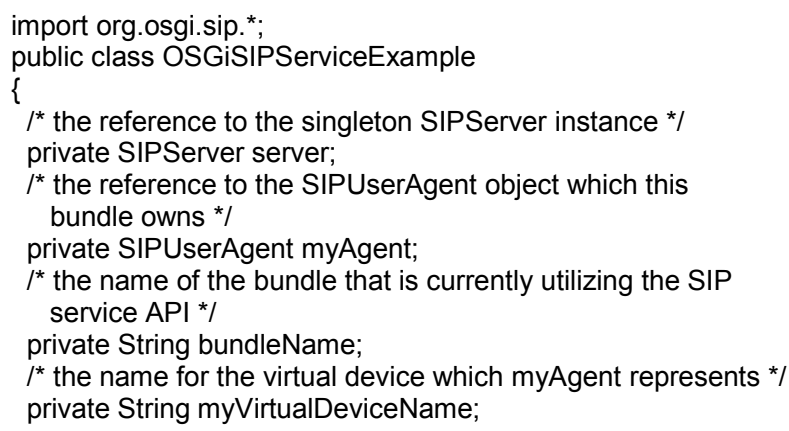

$/^{*}$ creates a SIPUserAgent object which this bundle can utilize to access the SIP functionalities. The created SIP user agent will be automatically registered with the SIP server and be treated as a "virtual SIP device". */

myAgent $=$ server .createSIPUserAgent $($ myVirtualDeviceName, bundleName, "virtual");

${ }^{*}$ creates an instance of DeviceMessageHandler and attaches it to the SIPUserAgent object. When an incoming SIP message arrives, the processRequest or processResponse method of messageHandler is invoked. In those two methods the bundle can conduct its own processings of the received SIP messages. */

DeviceMessageHandler messageHandler $=$ new DeviceMessageHandler(); myAgent.addSipListener(messageHandler);

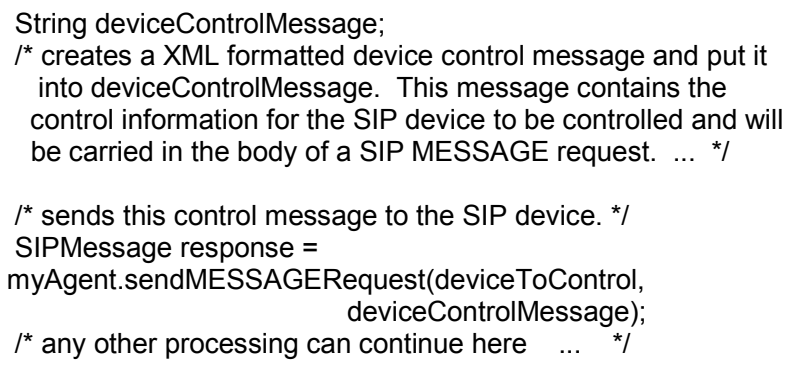

\section{Conclusions and Future Work}

In this paper, we proposed a novel OSGi-compliant SIP Service, which enables device and service mobility, crossimportation of OSGi devices and services, and OSGi support for SIP-based devices. The OSGi Forum Core Platform Expert Group (CPEG) is now working out the details of the SIP Service API.

\section{References}

[1] P. Dobrev, D. Famolari, C. Kurzke, "Device and Service Discovery in Home Networks with OSGi", Communication Magazine, IEEE Communication Society, pp. 86-92, New York, August 2002.

[2] S. Moyer, D. Maples, S. Tsang, A. Ghosh, "Service Portability of Networked Appliances", Communication Magazine, IEEE Communication Society, New York, pp. 116-121, January 2002.

[3] J. Rosenberg, H. Schulzrinne, G. Camarillo, A. Johnston, J. Peterson, R. Sparks, M. Handley, and E. Schooler. RFC 3261 - SIP: Session Initiation Protocol. IETF, 2002.

[4] SIP Testbed at Columbia University Reports http://www.cs.columbia.edu/ hgs/research/IRT/

[5] H. Schulzrinne, E. Wedlund, "Application-layer Mobility using SIP", Mobile Computing and Communications Review, Vol 1, No. 2, 2000.

[6] J. Undery, "Enhanced Usage of HTTP Digest Authentication for SIP", draft-undery-sip-auth01.txt, www.ietf.org, IETF, 2003. 
[7] Capability Negotiation Using SIP, Fujitsu Internet Draft: http://www.softarmor.com/sipwg/drafts/ morgue/draft-nishigaya-sip-ccpp-00.txt, IETF 2000.

[8] SIMPLE (SIP for Instant Messaging and Presence Leveraging Extensions), http://www.ietf.org/html.charters/simplecharter.html, IETF 2000.

[9] Third-generation Partnership Project (3GPP): http://www.3gpp.org/

[10]Device Control Using SIP, Telcordia: http://www.agreenhouse.com/iapp

[11]Open Service Gateway Interface Specification, Release 3, www.osgi.org

[12]Universal Plug and Play, www.upnp.org

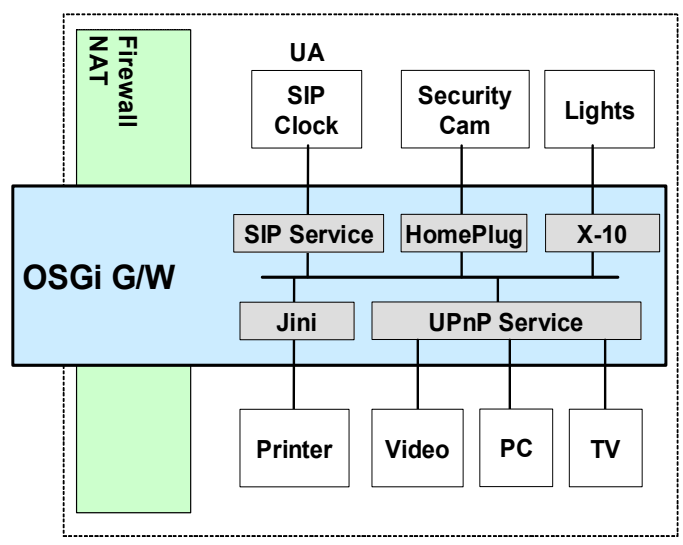

Figure 1. OSGi Home Gateway

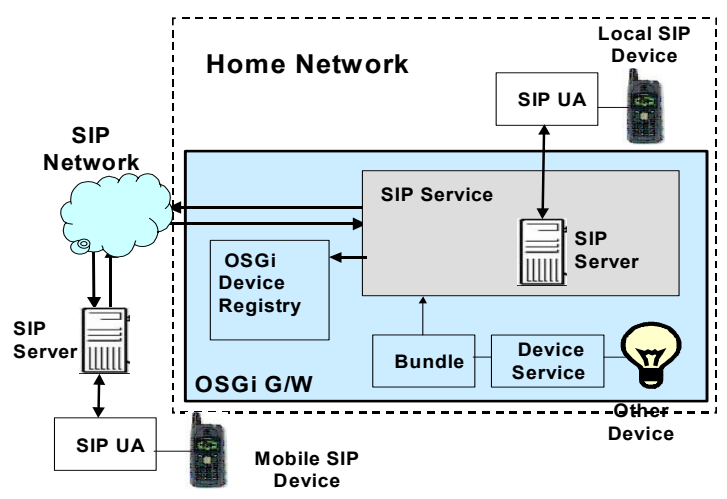

Figure 2. SIP Service Supported Architecture

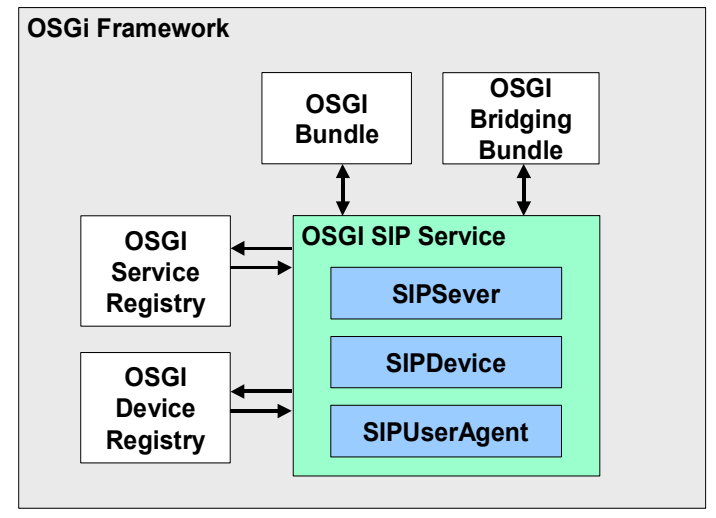

Figure 3. SIP Service Hierarchy

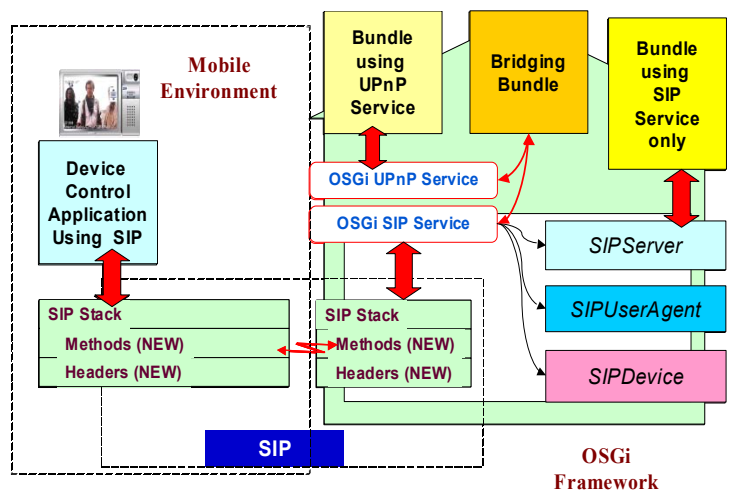

Figure 4. Bridging Bundles and their Role

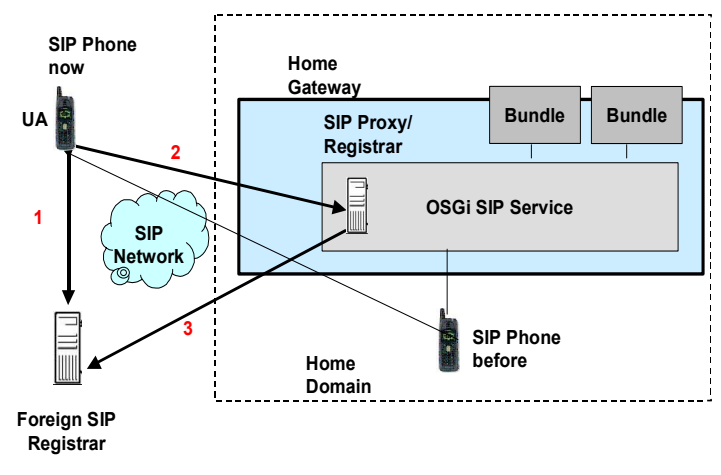

Figure 5. SIP Device Mobility and SIP Service 